\title{
Spatial heterogeneity in distribution and ecology of Western Palearctic birds
}

\author{
A. P. Møller,${ }^{1,2,5}$ J. J. Soler, ${ }^{3}$ And M. Martín Vivaldi ${ }^{4}$ \\ ${ }^{1}$ Laboratoire d'Ecologie, Systématique et Evolution, CNRS UMR 8079, Université Paris-Sud, \\ Bâtiment 362 F-91405 Orsay Cedex, France \\ ${ }^{2}$ Center for Advanced Study, Drammensveien 78, NO-0271 Oslo, Norway \\ ${ }^{3}$ Departamento de Ecología Funcional y Evolutiva, Estación Experimental de Zonas Aridas (CSIC). \\ General Segura 1, E-04001 Almería, Spain \\ ${ }^{4}$ Departamento de Biología Animal, Universidad de Granada, E-18071 Granada, Spain
}

\begin{abstract}
Species vary in abundance and heterogeneity of spatial distribution, and the ecological and evolutionary consequences of such variability are poorly known. Evolutionary adaptation to heterogeneously distributed resources may arise from local adaptation with individuals of such locally adapted populations rarely dispersing long distances and hence having small populations and small overall ranges. We quantified mean population density and spatial heterogeneity in population density of 197 bird species across 12 similarly sized regions in the Western Palearctic. Variance in population density among regions differed significantly from a Poisson distribution, suggesting that random processes cannot explain the observed patterns. National estimates of means and variances in population density were positively correlated with continental estimates, suggesting that means and variances were maintained across spatial scales. We used Morisita's index of population abundance as an estimate of heterogeneity in distribution among regions to test a number of predictions. Heterogeneously distributed passerine bird species as reflected by Morisita's index had small populations, low population densities, and small breeding ranges. Their breeding populations had been consistently maintained at low levels for considerable periods of time, because the degree of genetic variation in a subsample of non-passerines and passerines was significantly negatively related to heterogeneity in distribution. Heterogeneously distributed passerine species were not more often habitat specialists than homogeneously distributed species. Furthermore, heterogeneously distributed passerine species had high annual adult survival rates but did not differ in annual fecundity from homogeneously distributed species. Heterogeneously distributed passerine species rarely colonized urban habitats. Finally, homogeneously distributed bird species were hosts to a greater diversity of blood parasite species than heterogeneously distributed species. In conclusion, small breeding ranges, population sizes, and population densities of heterogeneously distributed passerine bird species, combined with their low degree of genetic variability, and their inability to colonize urban areas may render such species particularly susceptible to human-influenced global climatic changes.
\end{abstract}

Key words: band-sharing coefficient; blood parasites; dispersal; fragmentation; Morisita's index; spatial scale; specialization.

\section{INTRODUCTION}

Interactions between living organisms and their environments are central to ecology and evolution. Species almost invariably occur abundantly in a few places, while they are rare in most places (Anscombe 1948, Taylor et al. 1978). A characteristic feature of the spatial distribution of most species on most spatial scales is patchiness (e.g., Taylor 1961, Brown 1995). The negative binomial is the most widely applied model to describe this heterogeneity, with the variance increasing positively and curvilinearly with the mean (Routledge

Manuscript received 23 July 2009; revised 17 December 2009; accepted 21 December 2009. Corresponding Editor: F. S. Dobson.

${ }^{5}$ E-mail: anders.moller@u-psud.fr and Swartz 1991), and Taylor's power law fits well to empirical abundance data (Taylor et al. 1978). Such heterogeneity in distribution may affect range size, total abundance and effective population size with important, long-term evolutionary consequences (e.g., Soler et al. 2009). Although patchy distributions are well known in the ecological literature, the factors contributing to such heterogeneity and their ecological consequences are less clear. Species may show patchy geographical distributions for a number of different reasons. It is the objective of the present study to attempt to identify the characteristics of species with heterogeneous distributions and assess the ecological and life history consequences of such heterogeneity. 
The null hypothesis of heterogeneity in distributions is that heterogeneity in population density follows a random Poisson distribution. If the null expectation does not account for observed patterns of population density, nonrandom dispersal among sites, and nonrandom spatial distribution of fecundity, survival, and recruitment may contribute to heterogeneity in distributions. Hence, the environment influences the demography of populations in the landscape, with species with high fecundity and low survival tracking changes in the environment more closely than species with low fecundity and high adult survival rate (e.g., Rosenzweig 1995, Begon et al. 1996). Species that can respond more quickly to environmental change will have high fecundity and low survival and hence be more broadly distributed within their current range, but also be better able to track changes in the environment that promote range expansion or contraction depending on changes in distributions of limiting resources. Such ability to track changes in the environment should include a long maximum dispersal distance, the production of a large number of propagules (and hence high fecundity), and rapid population turnover due to low adult survival.

Spatial heterogeneity in distribution is caused by fragmentation. Spatial heterogeneity is crucial for understanding evolutionary processes including speciation (Roff 1997, Fahrig 2003). For example, small populations run an elevated risk of local extinction (e.g., Hanski 1999), and loss of genetic variation is more rapid in subdivided populations (e.g., Roff 1997). While natural habitat fragmentation is common, human impact on this ecological process is enormous and has increased rapidly during recent decades. Thus, fragmentation has been of particular concern from ecological, evolutionary and conservation perspectives because human impact on ecosystems affects the geographical distribution of species-specific habitats and thereby connectivity between individuals within species inhabiting them (e.g., Saunders et al. 1991, Andrén 1994, Turner 1996, Fahrig 1997, McGarigal and Cushman 2002).

Here we attempt to provide a general overview of the relationships between levels of heterogeneity in distributions of species at a large geographic scale and important species-specific traits related to susceptibility to environmental change, population connectivity, and local adaptation (Fig. 1a). Independently of whether heterogeneity in distributions of species is the cause or the consequence of such relationships, most predictions that we present here are well supported by theoretical and empirical findings. Therefore, we use this general framework for a number of statistical tests.

There is enormous variation in range size among species, varying from local endemics to widespread cosmopolitan species occurring everywhere (Gaston 2003). Range size has important implications for total population size because a large range may provide resources for a large population, although that is not always the case. Species that are common locally also tend to be common on a larger geographic scale, and, therefore, local and global population density is generally positively correlated (review in Brown and Lomolino 1998). An exception to this general rule is that habitat specialization in birds has been observed to be associated with both low local and global population density, with the caveat that specialists are relatively more common when the rest of the community is also specialized (Julliard et al. 2006). Thus, if local population density is low for heterogeneously distributed species, and if habitat specialists have low global population densities, heterogeneously distributed species will have low local and overall population sizes (Fig. 1b). Specialists may, through their stricter habitat requirements, also have more narrow ranges than generalists (Fig. 1b).

Heterogeneous distributions may arise from low levels of evolutionary specialization to heterogeneous environments. Indeed, generalist species that are able to manage successfully in a range of habitats tend to have wide niches, while species that are specialists only succeed in a narrow range of habitats that may be locally, but not globally abundant (Levins 1962, MacArthur 1972, Futuyma and Moreno 1988, Julliard et al. 2006). Recently, Devictor et al. (2008) showed that specialist species of birds had a more negative response to landscape fragmentation in accordance with the predicted positive relationship between heterogeneous distribution and specialization (Fig. 1c). A central theme of specialization is the high degree of exploitation of single resources. Because species with more heterogeneous distributions are predicted to be more specialized than homogeneously distributed species, but also to be more restricted in distribution and to have smaller population sizes, heterogeneity in distributions may provide the link between these species-specific traits (Fig. 1b).

Species with a limited dispersal propensity should produce few competitively superior offspring with a high probability of survival, while species with greater dispersal propensity should produce many offspring of poorer competitive ability (e.g., Levins 1962, 1968, MacArthur 1972, Roff 1997; Fig. 1b). Heterogeneous distribution may relate to habitat specialization, dispersal and life history. A high degree of specialization to local environmental conditions caused by narrow niches allows efficient exploitation of a few resources (Levins 1962, MacArthur 1972, Futuyma and Moreno 1988). Such specialization on the local environment (Fig. 1c) generally results in lower dispersal abilities (Tripet et al. 2002, Brouat et al. 2004; Fig. 1c), which in turn may affect the level of genetic variation (e.g., Roff 1997, Clobert et al. 2001; Fig. 1c). Reduced dispersal will invariably result in increased intensity of intraspecific competition (Dall and Cuthill 1997, Clobert et al. 2001). Dispersal plays a central role in maintaining local populations in a meta-population (Hanski 1991). 
a

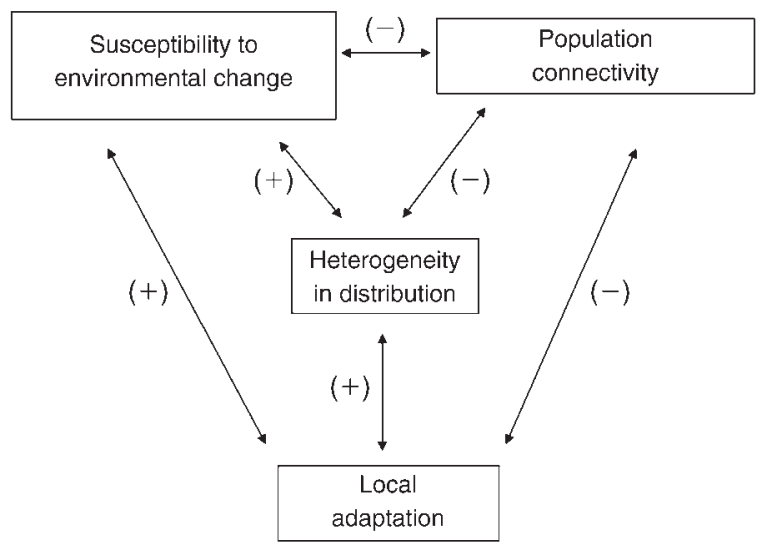

b) Local adaptation

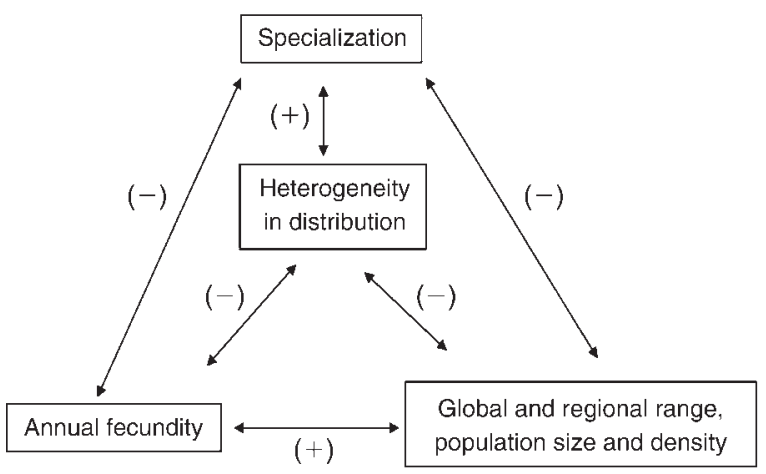

c) Population connectivity

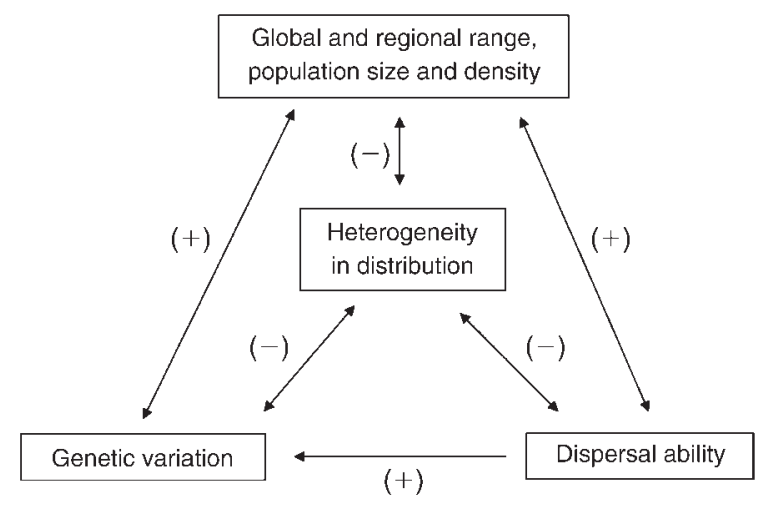

d) Susceptibility to environmental change

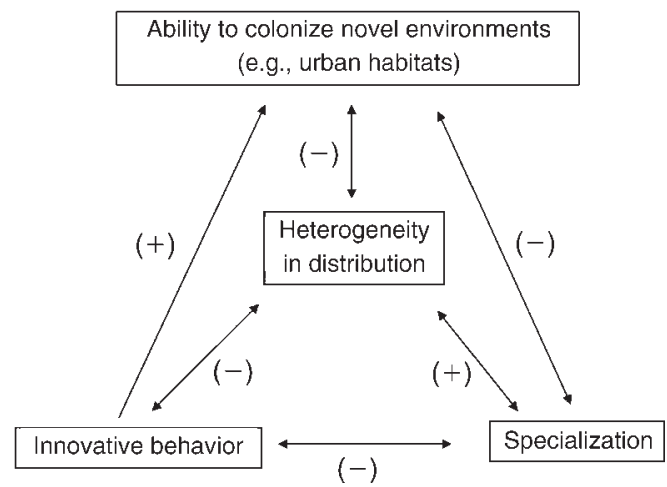

FIG. 1. (a) Hypothetical relationships between heterogeneous distribution and population connectivity, susceptibility to environmental change, and local adaptation. The set of variables hypothesized to be implied in each of these three scenarios is explained in detail in panels (b), (c), and (d), respectively. Relationships between different factors included in the present study are shown by arrows, with the direction of the effects being indicated by $(+)$ for a positive effect or $(-)$ for a negative effect.

Because local extinction probability increases with decreasing local population size (Hanski 1991), heterogeneous distributions arise as a consequence of dispersal being unable to rescue local populations. Therefore, more migrants and higher levels of immigration lead to more occupied patches and hence larger local and global populations (Hanski et al. 1995). These variables should be related to the level of heterogeneity in distribution of different species.

Heterogeneous distributions may affect the ability to colonize novel environments such as urban habitats (e.g., Møller 2009). Reduced colonizing ability may affect the frequency of innovative behavior that would allow individuals to exploit novel resources in new environments (Sol et al. 2002). Indeed, Sol et al. (2002) have shown that bird species with high rates of feeding innovations are better colonizers during human introductions than species with low rates of innovation (Fig. 1d). Similarly, Møller (2009) has shown that successful invasion of urban environments by birds is associated with a high rate of feeding innovations that may allow exploitation of novel food sources (Fig. 1d). Therefore, we predicted a negative relationship between heterogeneity of distribution and successful colonization of novel environments such as urban habitats, partly because of reduced dispersal ability.

Parasites may differ in their ability to exploit hosts depending on the degree of homogeneity in distribution of their hosts. Parasites that rely on access to evenly distributed hosts will have greater difficulty of maintaining viable populations in hosts that are heterogeneously distributed. That should particularly be the case for specialist parasites that can only survive on a single host. Such specialist parasites tend to be more virulent than generalist parasites (Combes 2001), and hosts have evolved stronger and more costly antiparasite defenses towards such parasites (Møller et al. 2005). Recently, Soler et al. (2009) have shown that host races of the brood parasitic European cuckoo Cuculus canorus exclusively have evolved in bird host species with homogeneous breeding distributions. Here, we tested whether hemosporidian blood parasites that included malarial parasites have diversified more and become 
TABle 1. Area $\left(\mathrm{km}^{2}\right)$ of different European countries and delimitation of regions used for analyses of the coefficient of variation $(\mathrm{CV})$ in population density in passerine birds from the Western Palearctic.

\begin{tabular}{|c|c|c|}
\hline Country & Area $\left(\mathrm{km}^{2}\right)$ & Region \\
\hline Portugal & 92389 & 1 \\
\hline Spain & 497446 & 1 \\
\hline Andorra & 468 & 2 \\
\hline France & 543965 & 2 \\
\hline Austria & 83858 & 3 \\
\hline Italy & 301225 & 3 \\
\hline Liechtenstein & 160 & 3 \\
\hline Switzerland & 41293 & 3 \\
\hline Albania & 28748 & 4 \\
\hline Bulgaria & 110994 & 4 \\
\hline Croatia & 56691 & 4 \\
\hline Greece & 131994 & 4 \\
\hline Slovenia & 20256 & 4 \\
\hline Hungary & 93033 & 5 \\
\hline Moldova & 33700 & 5 \\
\hline Romania & 237500 & 5 \\
\hline Czech Republic & 78864 & 6 \\
\hline Poland & 312685 & 6 \\
\hline Slovakia & 49036 & 6 \\
\hline Belgium & 30528 & 7 \\
\hline Germany & 356974 & 7 \\
\hline Luxembourg & 2586 & 7 \\
\hline Netherlands & 41526 & 7 \\
\hline Ireland & 70285 & 8 \\
\hline United Kingdom & 244110 & 8 \\
\hline Denmark & 43094 & 9 \\
\hline Norway & 323878 & 9 \\
\hline Sweden & 449964 & 10 \\
\hline Belarus & 207600 & 11 \\
\hline Estonia & 45226 & 11 \\
\hline Latvia & 64600 & 11 \\
\hline Lithuania & 65301 & 11 \\
\hline Finland & 338145 & 12 \\
\hline
\end{tabular}

more specialized in hosts with a homogeneous breeding distribution.

Taking into account the predictions described above, we used natural variation in heterogeneity of distribution of Western Palearctic bird species to test for the presumed ecological and evolutionary correlates of such heterogeneity (Fig. 1). We focused our study on passerine species, although we also included nonpasserines when testing the prediction concerning the relationship between genetic variation and heterogeneity of distribution because of small sample sizes. The predicted relationships and their scientific foundations are described above.

Before testing the predictions, we considered the question whether there is any evidence that the observed patterns of mean and variance in population density deviate from null expectations. If random processes accounted for patterns of density, we should expect densities to follow a Poisson distribution, with the variance $V$ equaling the expectation $E$. Thus, the ratio $V / E$ should equal one, if a Poisson distribution accounted for the interspecific patterns of population density. We only have cause to test additional predictions if this null model is rejected. We test and reject this null model, and continue by testing a number of predictions as outlined above.

\section{Methods}

\section{Population density}

Estimation of mean population density and heterogeneity in density was based on information from Tucker and Heath (1994) and Hagemeijer and Blair (1997) for a total of 199 species that was reduced to 170 species due to missing values for the variables included in the analyses. Maximum and minimum estimates of the number of breeding pairs in 33 countries (Table 1) were derived from Tucker and Heath (1994). Information for countries not present in Tucker and Heath (1994; Bosnia, Macedonia, Serbia) was obtained from Hagemeijer and Blair (1997). We checked the breeding distribution maps in Hagemeijer and Blair (1997) to confirm absence or presence of the target species and checked the list of the 10 countries with the largest populations. If some of these 10 countries were not included in Tucker and Heath (1994), we estimated minimum and maximum values directly from the numbers in Hagemeijer and Blair (1997). In addition, minimum and maximum value reported for the group "other countries" in Hagemeijer and Blair (1997) was used for estimating values for countries that were not included in the top-ten list, but where a target species was present. The remaining breeding pairs were assigned proportionally to the different countries according to their area after subtracting maximum and minimum population density for countries for which we collected data from Tucker and Heath (1994), but countries that were absent from the top-10 list and assigned to "other countries" in Hagemeijer and Blair (1997).

Measurement error is a potential cause of concern because such error may vary systematically with distribution or abundance. We used two independent estimates of population density of birds in Spain from Hagemeijer and Blair (1997) and Martí and del Moral (2003) to test for consistency in measurement error. We split the data into two categories: above and below the median abundance in Spain, and above and below the median distribution in Spain (i.e., variance in abundance of birds), from these two sources of data. We estimated repeatability of $\log _{10}$-transformed population density, using the equations in Becker (1984). Repeatability $(R)$ below the median abundance was $0.64(\mathrm{SE}=0.10), F=$ $4.55, \mathrm{df}=65,66, r^{2}=0.82, P<0.0001$, while it was 0.71 $(0.08)$ above the median $\left(F=5.97, \mathrm{df}=65,66, r^{2}=0.85\right.$, $P<0.0001)$. These two repeatability estimates are not significantly different. Repeatability $(R)$ with respect to distribution showed a similar pattern (below the median: $R(\mathrm{SE})=0.70$ (0.09), $F=5.61, \mathrm{df}=62,63, r^{2}=0.85, P<$ 0.0001 ; above the median: $R(\mathrm{SE})=0.84(0.05), F=$ $\left.11.61, \mathrm{df}=62,63, r^{2}=0.92, P<0.0001\right)$. Thus, there was no evidence suggesting that measurement errors varied consistently with respect to abundance or distribution. 
Heterogeneity in distribution is usually estimated by using indices of aggregation such as Morisita's index or other more traditional measures of spatial distribution based on the variance to mean ratio (Hurlbert 1990). Distribution range of species is also a measure of spatial aggregation of individuals and, consequently distribution range and heterogeneity in distribution are inevitably related (Hartley 1998). Thus effects of range and heterogeneity in distribution on target dependent variables can be studied by including indices of both these factors in linear models as predictor variables. Because of the relationship between heterogeneity in distribution and geographic range mentioned above, the use of samples (i.e., regions) where a target species is absent will provide important information, not only for the estimation of geographic range, but also for the estimation of heterogeneity indices. Rare species are predicted to be more clumped than common species (Cornell 1982), and, therefore, have more heterogeneous distributions. Therefore, when comparing values of heterogeneity in distribution of species considering similar geographic range of sampling for all species considered, the number of sampling areas where a target species is absent is important for estimation of indices of heterogeneity. Thus, the absence of a species from a geographic area will be related to geographic range and heterogeneity in distribution. However, by including geographic range and the number of sampling areas where a target species is absent as covariates we statistically control for this association while using all areas within the Western Palearctic geographical range for estimation of heterogeneity.

With the minimum and maximum population size values we estimated geometric means for each country as $\exp ([(\log [\operatorname{minimum}+1]+\log [\operatorname{maximum}+1]) / 2]-1)$ which is appropriate for data of exponential nature as is the case for population size. Density for each country was thus estimated as geometric means divided by country area in square kilometers, and population size was subsequently used for calculating Morisita's index.

Estimates of population size for each country were largely dependent on the area of different countries, and in an attempt to standardize our estimates to variation in area of countries we defined 12 different geographic regions of approximately similar size (Table 1). For each of these 12 geographic regions we summed population sizes of different countries (geometric means), as well as the area of each country within a given region. Afterward, we estimated population density as population size divided by area of each region.

Finally, for each species, we estimated means, standard deviations, and sums of population geometric means of population density among regions. With these values we estimated Morisita's index (Imor) for heterogeneity in population density, which by definition reflects variance in population density of different areas standardized for mean population density (Hurlbert 1990), using the following equation:

$$
\text { Imor }=n \times\left(\operatorname{sum}\left[x_{i}^{2}\right]-\operatorname{sum}\left[x_{i}\right]\right) /\left(\operatorname{sum}\left[x_{i}\right]^{2}-\operatorname{sum}\left[x_{i}\right]\right)
$$

where $x_{i}$ is the species abundance and $n$ is the number of European regions $(i=1,2, \ldots, 12)$.

Thus, Morisita's index varied between 0 and the number of defined European regions and high and low index values would indicate relatively heterogeneous and homogeneous distributions, respectively.

We compared Morisita's index as an estimate of heterogeneity in distribution to the coefficient of variation in population density among countries. Indeed, Morisita's index of heterogeneous distribution was strongly positively related to the coefficient of variation (Pearson $r=0.98, t=65.98, P<0.0001, N=$ 197). We used Morisita's index because it is preferable to traditional indices on theoretical grounds (Hurlbert 1990).

Furthermore, reliability of our estimates of heterogeneity of distributions was tested by means of repeatability analyses. First, we estimated repeatability of values for different countries within the same region. Briefly, for each of the nine Western Palearctic regions with more than one country we selected the two countries with the largest area. Then, we estimated Morisita's index for species abundance of these nine regions for all analyzed species taking into account countries with the largest and the second largest area within each region. Finally, repeatability $(R)$ of these values for species that appeared in both data sets was estimated by means of one-way ANOVAs $(R=0.68, F=$ $5.24, \mathrm{df}=174,175, P<0.0001)$ and was significantly repeatable. These results indicate that estimations for our 12 Western Palearctic regions were not influenced by identity of countries within a given region or any associated difference (e.g., countries using different sampling methods or effort), and they validate the use of mean values for each region.

Second, to rule out the possibility that our estimates depended on the Western Palearctic regions included in the analyses, we estimated Morisita's index of population abundance for each species by taking into account separately only regions with even or odd identification numbers in Table 1. Finally, we used one-way ANOVAs to estimate repeatability of this index estimated for different species separately using data from the two groups of Western Palearctic regions. Again, density for all analyzed species was significantly repeatable $(R=$ $0.71, F=6.00, \mathrm{df}=183,184, P<0.0001)$. These results indicate that our estimates of population density and heterogeneity do not depend on the Western Palearctic regions for which data were collected, and, consequently, our estimates can be considered species-specific characteristics.

\section{Testing for scale-dependent population density}

We tested explicitly if estimates of variation in population density across the Western Palearctic reflected variation at a more local scale by comparing 
variances among European regions with independent estimates of variation in population density from Denmark and Spain. We recorded the population density for the 43 species of common breeding passerine birds on the Danish mainland, by using scanned versions of the maps of population densities (Grell 1998). Grell (1998) published maps of the density of breeding birds based on systematic point count censuses of breeding birds carried out by hundreds of amateurs during 1994-1998 in all $5 \times 5 \mathrm{~km}$ squares throughout Denmark. These point counts provide reliable estimates of breeding bird density as shown by extensive analyses of potential sources of error and bias (see summary in Grell 1998).

We scanned the Danish maps for the 43 passerine species and estimated mean population density. We overlaid a $3 \times 3 \mathrm{~km}$ grid, and sampled the color of the maps of population density (using the RGB analysis plug-in) within each square in the grid using Adobe Photoshop. We then digitized the density scale from each map (species specific) and plugged these into a multiple regression to develop an equation relating color to density. Then, using a multiple regression equation, we calculated a density for each point sampled, for each map. An analysis of repeatability for 100 points that were digitized three times revealed a highly significant model $\left(F=846.50\right.$, df $=89,180, r^{2}=0.998, P<0.0001$, $R$ [repeatability] $=0.997, \mathrm{SE}=0.0009)$. The total number of density estimates per species ranged from 4100 to 4300 .

Martí and del Moral (2003) report the number of $10 \times$ $10 \mathrm{~km}$ squares of Spain (total squares: 5571) within each of the interval values of population density on a $\log _{10}$ scale. We assumed central values for each of the intervals, and estimated means and variances in population density, and Morisita's index of the 134 bird species coincident with those in our European data set.

\section{Population size}

Population sizes were obtained from Hagemeijer and Blair (1997), who reported the total number of breeding pairs in the Western Palearctic west of the Ural Mountains, obtained in a consistent way from national bird census programs in all countries. We used the arithmetic mean of the minimum and maximum estimates.

\section{Range size}

We determined the global northernmost and southernmost latitude of the breeding and the wintering distributions, respectively, to the nearest tenth of a degree of all species. Breeding range was the northernmost minus the southernmost breeding latitude, while wintering range was the northernmost minus the southernmost winter latitude. Information on breeding and wintering ranges was obtained from Cramp and Perrins (1988-1994). These estimates have previously been shown to provide biologically meaningful measures of distribution (Gaston and Blackburn 1996).

We also estimated breeding range in the Western Palearctic and total breeding range as the area of the shape bounded by the greatest span of latitude and longitude of each species' breeding range, as published in Cramp and Perrins (1988-1994). To take into account the curvature of the earth (which was assumed to be spherical), this area was estimated by the following equation:

$$
\begin{aligned}
\text { Area }= & \mathrm{RE}^{2} \times\left(\text { longitude }_{1}-\text { longitude }_{2}\right) \\
& \times\left(\sin \left[\text { latitude }_{1}\right]-\sin \left[\text { latitude }_{2}\right]\right)
\end{aligned}
$$

where RE is the radius of the earth $(6366.2 \mathrm{~km})$ and latitude and longitude are expressed in radians.

In widespread species Old and New World ranges were calculated separately and subsequently summed. This method overestimates true geographical range because parts of the range that is unoccupied are also included, although this error should be random with respect to the variables of interest. Estimates of area were strongly positively correlated with geographical range size as calculated by counting one-degree grid cells overlain on published distribution maps for a sample of 20 Palearctic and Nearctic bird species $(r=0.87, P<$ $0.001)$, and with range size as reported for a sample of 11 threatened species (Stattersfield and Capper 2000; $r=$ $0.98, P<0.001$, based on log-transformed data).

\section{Habitats and habitat specialization}

We used the habitat specialization index as defined by Julliard et al. (2006). Briefly, this index was based on the variance in average breeding population densities among 18 habitat classes in France, by calculating the coefficient of variation in population density among these 18 habitat classes.

We defined a species as being urbanized based on information in Cramp and Perrins (1988-1994) and Glutz von Blotzheim and Bauer (1966-1997). A species being classified as urbanized had to fulfill the following criteria, following Møller (2009): (1) breeding populations occur inside towns and cities and (2) population densities in towns and cities are higher than in rural habitats.

\section{Fecundity}

We recorded clutch size and maximum number of clutches per season, allowing us to estimate annual fecundity (clutch size multiplied by maximum number of clutches per season) from Cramp and Perrins (19881994). If multiple estimates were provided, we extracted the information from the UK because those estimates were generally based on the largest sample sizes. We also extracted mean body mass of males and females during the breeding season from Cramp and Perrins (19881994), again generally preferring estimates from the UK due to larger sample sizes. Body mass was calculated as the mean of male and female body mass. 


\section{Survival rate}

We recorded annual adult survival rate from Cramp and Perrins (1988-1994). If multiple estimates were provided, we extracted the information from the UK because those estimates generally were based on the largest sample sizes.

\section{Band sharing}

The band-sharing coefficient is an estimate of the number of shared minisatellite bands in relation to the total number of bands among adults, originally proposed by Wetton et al. (1987) as a measure of genetic similarity. A high band-sharing coefficient implies that many bands are shared among individuals and hence that the genetic similarity in the population is relatively high. This estimate of band sharing has commonly been used as an index of genetic similarity among individuals within a species (e.g., Reeve et al. 1990, Hoelzel 1992, Blomqvist et al. 2002). Here, we use species-specific mean band-sharing values for comparative purposes. This approach is supported firstly by the highly significant repeatability (Becker 1984) of band-sharing coefficients among studies of the same species using the same probe, based on 25 studies of nine species $(R=0.55$ $[\mathrm{SE}=0.20], F=3.90, \mathrm{df}=8,16, P=0.0099$, see also Møller [2001] and Spottiswoode and Møller [2004]). Secondly, Papangelou et al. (1998) have shown in a review of 129 published studies of 70 bird species that mean band-sharing coefficient between dyads of unrelated individuals from populations or species defined as outbred on the basis of independent evidence was nearly $50 \%$ of that for populations defined as small or inbred. In addition, mean band sharing between dyads of unrelated individuals in small or inbred populations was similar to that for dyads of first-order relatives in outbred populations (Papangelou et al. 1998). This implies that mean band-sharing coefficients of populations consistently reflect whether there is a high degree of genetic similarity within a species.

We estimated genetic similarity as the band-sharing coefficient between adults in a breeding population (usually dyads of pair members, but in cases where dyads of random adults in the population were also compared results were very similar). Thus band-sharing coefficients and total number of bands were recorded from local populations that at most covered a few square kilometers. The information derived from an extensive survey of the literature using the Web of Science (Thomson Reuters, Philadelphia, Pennsylvania, USA) as a source, and Spottiswoode and Møller (2004) report all references. However, we did not include estimates for populations that had recently gone through bottlenecks such as House Sparrows Passer domesticus from North America or the Great Reed Warbler Acrocephalus arundinaceus from Lake Kvismaren, Sweden.

Several factors may potentially cause heterogeneity in band-sharing estimates. The number of fingerprinting probes ranged from one to three per study (these probes were mainly 33.15 and 33.36 , but also per, M13, and others). This could potentially cause heterogeneity in estimates among analyses. Repeatability of band-sharing coefficients among different probes used in the same study, based on 47 studies of 20 species, was highly significant $(R=0.86[\mathrm{SE}=0.06], F=17.16, \mathrm{df}=19,27, P$ $<0.0001$; see also Møller [2001] and Spottiswoode and Møller [2004]), suggesting that multiple probes should not cause substantial bias. Finally, band-sharing coefficients were independent of the number of bands scored (Møller 2001).

Because band-sharing coefficients were only available for a small number of species, we included both nonpasserines and passerines in an attempt to increase the sample size and hence the power of the statistical test.

\section{Dispersal}

We estimated maximum dispersal distance as the minimum distance from the mainland to an island with a permanent breeding population (Møller and Mousseau 2007), using information from the distribution maps for passerine bird species in Cramp and Perrins (19881994). We did that by considering the distance from the mainland to all islands closer than the most distant island to the mainland with a permanent breeding population. Therefore, the estimate of maximum dispersal distance was a minimum estimate because many populations on islands are likely not to have taken the shortest route from the mainland to an island, and because islands may have been colonized directly rather than by using intermediate islands as stepping-stones. This estimate of maximum effective dispersal distance assumes that all species have the possibility to colonize the same set of islands. This and other assumptions are fully discussed in Møller and Mousseau (2007).

\section{Blood parasites}

Parasite species richness quantified as the number of parasite species was extracted from Peirce (1981) and Scheuerlein and Ricklefs (2004) combined with information from several sources listed in Møller and Nielsen (2007). Although molecular techniques may be better at detecting weak infections, several studies have shown a positive association between estimates of parasite prevalence using both microscopic and molecular techniques (e.g., Waldenström et al. 2004, Ricklefs et al. 2005). Our own analyses of the data provided in the supplementary material in Ricklefs et al. (2005) showed positive consistency among the two estimates of parasite prevalence (Kendall $\tau=0.307, z=6.252, N=188, P<$ 0.001 ), although analyses of blood smears only revealed $28 \%$ of what was found with PCR. Thus, analyses based only on microscopy are conservative. In total, the analyses presented here were based on infection levels of 29799 adult hosts belonging to 263 species. Finally, we extracted information on research effort as the number of individuals examined for each host species to 
control for the potentially confounding effect of sampling effort in the analyses.

The entire data set is reported in Appendix A.

\section{Statistical analyses}

All statistical tests were made with JMP (2000). Maximum dispersal distance, research effort, area of the total breeding range and the breeding range in the Western Palearctic, population size in the Western Palearctic, annual fecundity, body mass, species richness of blood parasites and research effort were $\log _{10^{-}}$ transformed before analysis, with a constant of 1 being added to maximum dispersal distance, while band sharing was square-root arcsine-transformed. Population density was analyzed by inclusion of range size and population size in the Western Palearctic as predictor variables. We tested for effects of violation of assumptions for parametric tests by reanalyzing all data using partial Kendall rank-order correlations. None of the conclusions changed as a consequence of this procedure suggesting that the conclusions were robust.

We tested the null hypothesis that the variance in population density was as expected from random processes by determining whether the variance in density $(V)$ differed from the expectation $E$, assuming that the ratio $V / E$ should approximate a value of one under a Poisson process. We used $\log _{10}$-transformed means and $\log _{10}$-transformed variances to normalize data before testing whether the ratio $V / E$ deviated significantly from a value of one.

We developed statistical models to quantify the role of heterogeneity in distribution as a determinant of ecological variables. All models were general linear models except invasion of urban environments that was analyzed using logistic regression. In the analyses we included Morisita's index, total range size and body mass as predictors because small species have higher densities, larger population fluctuations and different life histories than large species.

The statistical analysis of species richness of blood parasites was based on data with large differences in sampling effort. To control for differences in sampling effort we included $\log _{10}$-transformed sample size as an additional predictor variable in the statistical model, as we have done in previous analyses (Arriero and Møller 2008).

\section{Comparative analyses}

Species-specific data are not statistically independent due to similarity in phenotype among species caused by common descent. We controlled for such statistical dependence by calculating independent linear contrasts for each variable to test for associations between variables according to the method developed by Felsenstein (1985).

The composite phylogeny was based on Sibley and Ahlquist (1990) combined with additional information from other sources (Thomas et al. 2004, Aliabadian et al. 2006, Jønsson and Fjeldså 2006). The phylogeny is shown in Appendix B.

We adopted the software CAIC for estimating standardized linear contrasts (Purvis and Rambaut 1995). We used two series of tests based on different branch length. First, branches were assigned the same length, and, second, we used uneven branch lengths, assuming a gradual evolution model as implemented in the software by Purvis and Rambaut (1995). Both series of tests produced qualitatively similar results. We tested for violations of the statistical assumptions by regressing absolute values of standardized contrasts against their standard deviations (Garland et al. 1992). None of these tests revealed significant deviations, after sequential Bonferroni adjustment for multiple tests. Contrasts were analyzed by forcing regressions through the origin because the dependent variable is not supposed to have changed when the independent variable has not changed (Purvis and Rambaut 1995). Multiple regression models were used to control for the effects of confounding factors on the variables of interest, restricting these to species for which all information was available. Once we had identified significant predictors, we performed a new multiple regression by only using these predictor variables to increase sample size and hence statistical power of the test.

Sample sizes differed among tests because information on different variables was not available for all species.

\section{Results}

\section{Heterogeneity in distribution}

We tested the null hypothesis that variance in population density was simply caused by random Poisson processes by determining whether the variance in population density $(V)$ was similar to the null expectation $(E)$. However, the ratio $V / E$ was on average $1.733(\mathrm{SE}=0.017)$, thus being significantly larger than the expected value of one $(t=4.42$, df $=197, P<$ $0.0001)$. Thus, the variance was significantly greater than expected from a Poisson distribution, suggesting that processes other than random sampling influenced variation in population density.

Variance in population density was strongly positively related to mean density $\left(F=7435.12\right.$, df $=1,195, r^{2}=$ $0.974, P<0.0001$, slope [and SE] $=1.754$ [0.020]). This high degree of colinearity precluded use of mean and variance in the same analysis, and we thus used Morisita's index in all subsequent analyses because this index captures heterogeneity in density among areas (Hurlbert 1990). Morisita's index was negatively related to mean density $\left(F=79.94, \mathrm{df}=1,195, r^{2}=0.29, P<\right.$ 0.0001 , slope $=-1.604[0.179])$ and variance in density $(F$ $=48.67, \mathrm{df}=1,195, r^{2}=0.20, P<0.0001$, slope $=$ -0.748 [0.107]).

Heterogeneity in distribution, as reflected by Morisita's index, ranged from 1.14 (Bullfinch, Pyrrhula pyrrhula) to 12.00 (several species), with a mean $=5.43$ [0.26], median 4.22, $N=197$ species. 


\section{Testing for scale-dependent variation in population density}

There was a significant positive association between $\log _{10}$-transformed continent-wide estimates of mean density used here and $\log _{10}$-transformed mean population densities in Denmark $\left(F=16.78\right.$, df $=1,41, r^{2}=$ $0.14, P=0.029$, slope $=0.55[0.14])$ and in Spain $(F=$ 67.1, df $=1,132, r^{2}=0.34, P<0.0001$, slope $=0.58$ [0.07]). Furthermore, there was a significant positive association between the $\log _{10}$-transformed continentwide estimate of variance used here and $\log _{10}$-transformed variance in population density in Denmark $(F=$ 6.02, $\mathrm{df}=1,41, r^{2}=0.14, P=0.019$, slope $\left.=0.41[0.17]\right)$ and in Spain $\left(F=76.0, \mathrm{df}=1,132, r^{2}=0.37, P<0.0001\right.$, slope $=0.60$ [0.07]). In conclusion, estimates of average population density and variance in population density were weakly consistent across national and continental spatial scales.

In a second test based on information from Spain, we found a significant positive correlation between mean population density among $100-\mathrm{km}^{2}$ squares in the Spanish atlas from 2003 (Martí and del Moral 2003) and that estimated in the European Atlas from 1997 (Hagemeijer and Blair 1997) for Spanish birds $(F=$ 374.45 , df $=1,132, r^{2}=0.74, P<0.0001$, slope $=0.86$ [0.04]), which indicates consistent estimates of population density across different census and sampling periods.

\section{Heterogeneity in distribution, population density and range size}

Species with heterogeneous distributions as reflected by Morisita's index had small total breeding ranges (Fig. 2, Table 2). This conclusion was similar when excluding two outliers that were island endemics (Loxia scotica and Sylvia sarda; partial effect of heterogeneity in distribution for species-specific data, $F=36.20$, df $=1$, 161, $P<0.0001$, slope $=-0.046$ [0.008]). Similar conclusions were reached if these analyses were based on range size in the Western Palearctic rather than total range size. Likewise, analyses of phylogenetically independent contrasts provided similar conclusions.

Species with heterogeneous distributions had small breeding population densities as expected from the correlation between local and global density, with an additional significant effect of body mass (Table 2). Total population sizes in the Palearctic were also smaller in species with heterogeneous distributions. These conclusions were repeated in analyses of phylogenetically independent contrasts.

\section{Heterogeneity in distribution and dispersal}

Species with heterogeneous distributions had shorter dispersal distances than species with homogeneous distributions (Fig. 3, Table 2), with an additional significant effect of range size since species with large ranges dispersed long distances. A similar conclusion was reached in an analysis of contrasts.

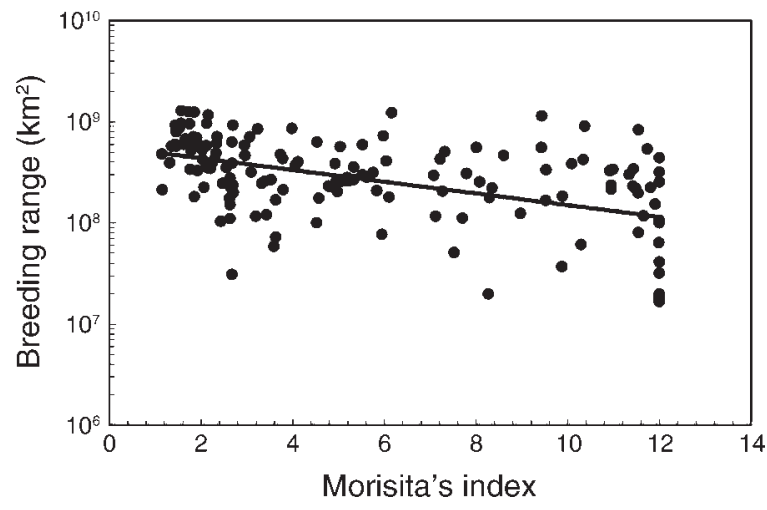

FIG. 2. Breeding range $\left(\mathrm{km}^{2}\right)$ in relation to Morisita's index among different Western Palearctic passerine bird species. The line is from a linear regression.

\section{Heterogeneity in distribution and genetic variation}

If species with heterogeneous distribution have maintained small populations for considerable periods of time, they should have a higher degree of band sharing. Analyses that included heterogeneity in distribution, range size and body mass showed a significant negative association between band sharing and Morisita's index in the species-specific analysis (Table 2). Variables in this model were redundant, and a best-fit model based on Akaike's Information Criterion for inclusion of variables revealed a model that included heterogeneity in distribution (Fig. 4; model $F=7.78$, df $=1,59, r^{2}=0.12, P=0.0071$, slope $=0.193$ [0.069]). For contrasts, the best model included effects of heterogeneity in distribution and body mass (model $F=4.15, \mathrm{df}=$ 2, 57, $r^{2}=0.07, P=0.021$; partial $F$ for heterogeneity in distribution $=4.08, \mathrm{df}=1,57, P=0.048$, slope $=0.136$ [0.067]; partial $F$ for body mass $=4.45, \mathrm{df}=1,57, P=$ 0.039 , slope $=-0.071[0.034])$.

\section{Heterogeneity in distribution and habitat specialization}

Heterogeneously distributed species did not differ significantly in terms of habitat specialization from homogeneously distributed species, with no additional significant effects of other variables (Table 2). A similar conclusion was reached in an analysis of contrasts.

\section{Heterogeneity in distribution and urban colonization}

Urban species were less heterogeneously distributed than non-urban species (mean Morisita's index [SE] for urban species $=3.09$ [0.43], $N=38$ species; mean for other species $=6.27$ [0.32], $N=131$ ), with an additional significant effect of range size (Table 2). This conclusion was not repeated for the analysis of contrasts, although exclusion of the nonsignificant variable body mass resulted in a significant relationship with Morisita's index $\left(F=4.15, \mathrm{df}=1,150, r^{2}=0.03, P=0.044\right.$, slope $=$ $0.020[0.010])$. 
TABLE 2. Relationships between heterogeneity in distribution as reflected by Morisita's index and various ecological variables after inclusion of range size and body mass as additional predictor variables.

\begin{tabular}{|c|c|c|c|c|c|c|c|c|}
\hline \multirow[b]{2}{*}{ Variable } & \multicolumn{4}{|c|}{ Morisita's index } & \multicolumn{4}{|c|}{ Range size } \\
\hline & Slope (SE) & $F$ & df & $P$ & Slope (SE) & $F$ & df & $P$ \\
\hline \multicolumn{9}{|l|}{ Species } \\
\hline Range size & $-0.054(0.009)$ & 34.21 & 1,163 & $<0.0001$ & & & & \\
\hline Range size in Western Palearctic & $-0.054(0.007)$ & 67.69 & 1,163 & $<0.0001$ & & & & \\
\hline Population density & $-0.058(0.018)$ & 10.08 & 1,162 & 0.002 & & & & \\
\hline Population size & $-0.124(0.017)$ & 51.21 & 1,163 & $<0.0001$ & & & & \\
\hline Band-sharing coefficient & $0.009(0.005)$ & 3.21 & 1,56 & 0.079 & $-0.091(0.040)$ & 5.17 & 1,56 & 0.027 \\
\hline Dispersal distance & $-0.038(0.014)$ & 6.87 & 1,162 & 0.0096 & $0.437(0.111)$ & 15.40 & 1,162 & 0.0001 \\
\hline Habitat specialization & $0.015(0.011)$ & 1.78 & 1,68 & 0.19 & $0.058(0.092)$ & 0.39 & 1,68 & 0.53 \\
\hline Urban colonization $\dagger$ & $0.270(0.088)$ & & & 0.002 & $-1.466(0.730)$ & & & 0.045 \\
\hline Annual fecundity & $-0.003(0.004)$ & 0.50 & 1,160 & 0.48 & $0.091(0.032)$ & 8.19 & 1,160 & 0.005 \\
\hline Adult survival rate & $0.018(0.006)$ & 10.24 & 1,70 & 0.0021 & $0.026(0.040)$ & 0.44 & 1,70 & 0.51 \\
\hline Number of blood parasite species & $-0.197(0.688)$ & 8.28 & 1,109 & 0.0048 & $1.163(0.688)$ & 2.86 & 1,109 & 0.09 \\
\hline \multicolumn{9}{|l|}{ Contrasts } \\
\hline Range size & $-0.052(0.009)$ & 65.65 & 1,150 & $<0.0001$ & & & & \\
\hline Range size in western Palearctic & $-0.050(0.006)$ & 62.45 & 1,150 & $<0.0001$ & & & & \\
\hline Population density & $-0.075(0.018)$ & 17.05 & 1,149 & $<0.0001$ & & & & \\
\hline Population size & $-0.139(0.017)$ & 64.91 & 1,150 & $<0.0001$ & & & & \\
\hline Band-sharing coefficient & $0.007(0.004)$ & 2.66 & 1,56 & 0.11 & $-0.058(0.034)$ & 2.94 & 1,56 & 0.09 \\
\hline Dispersal distance & $-0.031(0.015)$ & 4.17 & 1,149 & 0.043 & $0.581(0.128)$ & 20.67 & 1,149 & $<0.0001$ \\
\hline Habitat specialization & $0.012(0.012)$ & 0.90 & 1,67 & 0.35 & $-0.071(0.104)$ & 0.46 & 1,67 & 0.50 \\
\hline Urban colonization & $-0.019(0.010)$ & 3.73 & 1,149 & 0.05 & $0.177(0.083)$ & 4.54 & 1,149 & 0.035 \\
\hline Annual fecundity & $-0.001(0.004)$ & 0.04 & 1,147 & 0.85 & $0.074(0.031)$ & 5.92 & 1,147 & 0.016 \\
\hline Adult survival rate & $0.012(0.006)$ & 3.93 & 1,67 & 0.05 & $0.026(0.040)$ & 0.44 & 1,67 & 0.51 \\
\hline Number of blood parasite species & $-0.013(0.006)$ & 4.68 & 1,102 & 0.033 & $0.284(0.024)$ & 144.12 & 1,102 & $<0.0001$ \\
\hline
\end{tabular}

Note: Analyses were made separately for species-specific data and independent linear contrasts.

$\uparrow$ Chi-square was calculated for urban colonization because urban colonization was a dichotomous variable analyzed with logistic regression. Values were: Morisita's index $\chi^{2}=9.48$; range size $\chi^{2}=4.04$; body mass $\chi^{2}=1.34$.

Heterogeneity in distribution, fecundity, and survival

Heterogeneously distributed species did not differ significantly in fecundity from more homogeneously distributed species, after controlling for the significant effects of body mass and range size (Table 2). That was also the case in an analysis of contrasts.

Heterogeneously distributed species tended to have higher adult survival rate than homogeneously distributed species (Fig. 5, Table 2), after controlling for the

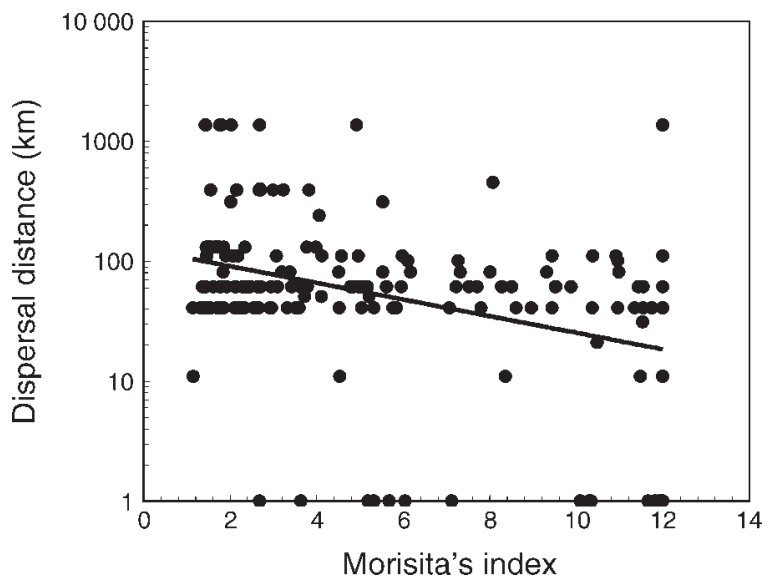

FIG. 3. Morisita's index in relation to maximum dispersal distance $(\mathrm{km})$ among different Western Palearctic passerine bird species. The line is from a linear regression. effects of body mass. That was also the case in an analysis of contrasts.

\section{Heterogeneity in distribution and blood parasites}

Species richness of blood parasites decreased significantly with increasing heterogeneity in distribution of their bird hosts (Fig. 6, Table 2), in a model that included sampling effort as an additional predictor variable. In the analysis of contrasts, there were additional effects of breeding range size and body mass. Therefore, species richness of blood parasites was the

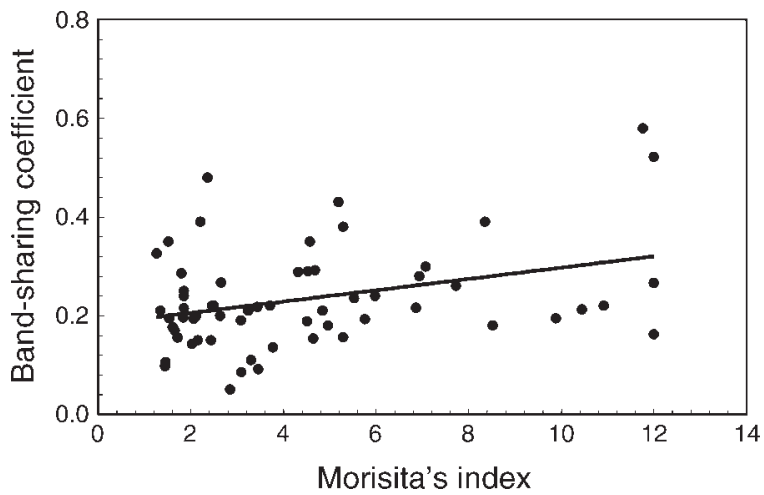

FIG. 4. Band-sharing coefficient in relation to Morisita's index among different Western Palearctic bird species. Bandsharing coefficients were square-root arcsine-transformed. The line is from a linear regression. 
TABLE 2. Extended.

\begin{tabular}{rrrc}
\hline \hline & \multicolumn{3}{c}{ Body mass } \\
\hline \multicolumn{1}{c}{ Slope (SE) } & $F$ & df & $P$ \\
\hline & & & \\
$0.128(0.086)$ & 2.14 & 1,163 & 0.14 \\
$0.029(0.061)$ & 0.23 & 1,163 & 0.63 \\
$-0.296(0.143)$ & 4.27 & 1,162 & 0.040 \\
$-0.261(0.161)$ & 2.62 & 1,163 & 0.11 \\
$-0.010(0.023)$ & 0.19 & 1,56 & 0.66 \\
$0.052(0.124)$ & 0.18 & 1,162 & 0.68 \\
$-0.104(0.062)$ & 2.80 & 1,68 & 0.10 \\
$-0.549(0.474)$ & & & 0.25 \\
$-0.200(0.035)$ & 32.67 & 1,160 & $<0.0001$ \\
$0.155(0.028)$ & 30.36 & 1,70 & $<0.0001$ \\
$0.135(0.482)$ & 0.08 & 1,109 & 0.78 \\
& & & \\
$0.266(0.200)$ & 1.76 & 1,150 & 0.19 \\
$0.103(0.148)$ & 0.49 & 1,150 & 0.49 \\
$-0.760(0.355)$ & 4.59 & 1,149 & 0.034 \\
$-0.630(0.399)$ & 2.49 & 1,150 & 0.12 \\
$-0.066(0.033)$ & 3.99 & 1,56 & 0.05 \\
$-0.067(0.315)$ & 0.04 & 1,149 & 0.83 \\
$0.068(0.154)$ & 0.20 & 1,67 & 0.66 \\
$-0.292(0.205)$ & 2.03 & 1,149 & 0.16 \\
$-0.268(0.075)$ & 12.76 & 1,147 & 0.0005 \\
$0.190(0.059)$ & 10.37 & 1,67 & 0.002 \\
$-0.122(0.058)$ & 4.34 & 1,102 & 0.040 \\
\hline
\end{tabular}

largest in bird hosts with homogeneous breeding distributions.

\section{Discussion}

This study of heterogeneity in population density at a continental scale showed variation in heterogeneity by almost an order of magnitude among species of passerine birds. Heterogeneously distributed species had small total and local breeding ranges and small overall population sizes and densities. These small populations have been maintained for long periods of time, as reflected by low levels of local genetic variation. Heterogeneously distributed species had short dispersal distances, and such species were less likely to colonize urban areas. Furthermore, heterogeneously distributed species had high adult survival rates. Finally, homogeneously distributed bird species had a greater diversity of blood parasite taxa than heterogeneously distributed species. These relationships are summarized in Fig. 1, which may be considered a tool for understanding the complex relationships between range, population size, and life history.

We found a strong positive correlation between means and variances in density among species, accounting for more than $97 \%$ of the variance. Colinearity between mean and variance implies that these two variables basically contain the same information, making it difficult to determine whether one or the other variable accounts for relationships between density and ecological variables. Here we used Morisita's index as a metric of heterogeneous distribution. We suggest that the general finding that local and global population sizes

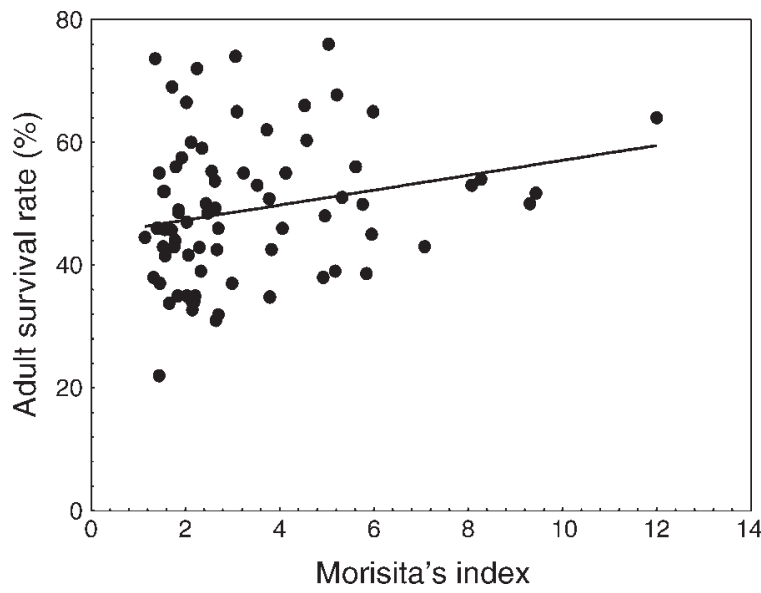

FIG. 5. Morisita's index in relation to annual adult survival rate among different Western Palearctic passerine bird species. The line is from a linear regression.

are positively correlated with each other (review in Brown and Lomolino 1998) can only be interpreted as implying that mean density, variance in density, or both account for specific relationships.

We have analyzed spatial patterns of population density both on a national scale and continental scales. We explicitly tested for differences in patterns of mean and variance in population density at different scales, using extensive bird census data from two countries (Denmark and Spain). We found positive correlations between mean population density at the national and the continental scale in both countries. Similarly, we found positive correlations between variances in population density at these two scales for both Denmark and Spain. Because local and global population densities are generally positively correlated (review in Brown and Lomolino 1998), this implies that we should find similar positive correlations between national and continental scales. That was indeed what we found.

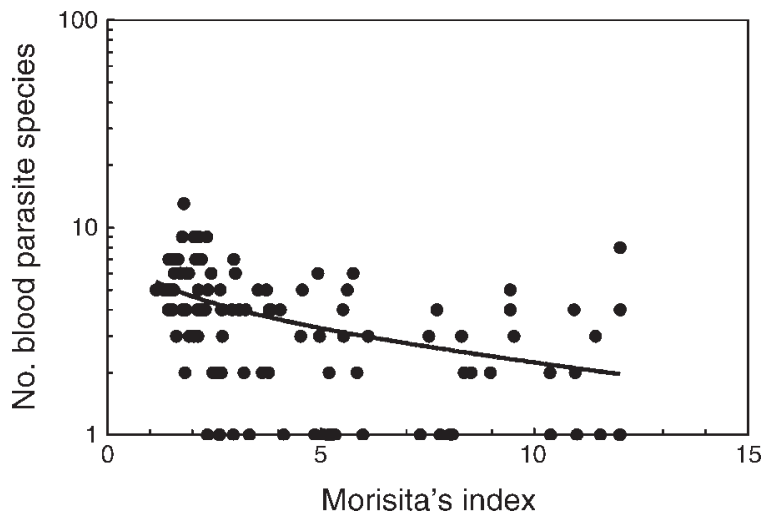

FIG. 6. Morisita's index in relation to species richness of blood parasites among different Western Palearctic passerine bird species. The line is from a linear regression. 
Many of the relationships investigated here had small to intermediate effect sizes (sensu Cohen 1988), typically accounting for $1 \%$ to $10 \%$ of the variance. Such levels of variance explained are typical in ecology and evolution, where scientists even with experimental approaches on average account for 5-7\% of the variance (Møller and Jennions 2002). We note that the negative relationship between range size and heterogeneity in population density (Fig. 2) is the null expectation, and that this negative association implies that covariation between ecological variables and heterogeneity in distribution may partly be accounted for by the effect of range size.

Heterogeneity in distribution among birds may be the consequence of natural fragmentation of habitats because certain species mainly occur in parts of their range, a range that is considerably smaller than that of homogeneously distributed species. This effect was not due to such species being habitat specialists because we found no evidence suggesting that habitat specialization was related to heterogeneity in population density. Previous studies have shown that habitat specialists do less well when co-occurring with generalists (Julliard et al. 2006), and that specialists are less able to cope with fragmentation than generalists (Devictor et al. 2008).

Dispersal and subsequent mating are the cause of gene flow within and among populations (Wright 1978, Slatkin 1987). We found that species of birds with heterogeneous breeding distributions have short maximum dispersal distances compared to more homogeneously distributed species, in accordance with expectations. This effect was independent of habitat specialization that was not significantly associated with heterogeneity in distribution. Although the level of local adaptation is usually high in habitat specialists (e.g., Møller et al. 2005), it can also be predicted that the intensity of local intraspecific competition is greater among specialists than generalists (e.g., Levins 1962, 1968, MacArthur 1972, Dall and Cuthill 1997) due to the clumped distribution of suitable resources and limited dispersal of the former species (Dall and Cuthill 1997, Tripet et al. 2002, Brouat et al. 2004).

We demonstrated that range size, population size and population density are all reduced in heterogeneously distributed species of passerine birds compared to sister taxa with homogeneous distributions. If these patterns have been maintained over long periods of time, this should have consequences for patterns of standing genetic variation. Here we used a measure of genetic variability among individuals in a local population. We have previously shown that this index is significantly repeatable among populations (Møller et al. 2008), implying that if one local population has a low level of genetic variability that is also likely to be the case for other local populations of the same species. Population subdivision could slow the overall loss of genetic variation in the total population of a species (e.g., Roff 1997). However, there is comparative evidence contrary to this suggestion because bird species with relatively more subspecies per unit area of distribution, and hence with a greater level of subdivision, actually have lower levels of genetic variation than species with fewer subspecies, even for a given breeding range, population size and body mass (Møller et al. 2008). Here we found that the band-sharing coefficient between unrelated adults in local populations was positively related to Morisita's index for population density. This implies that genetic variability was reduced in species with heterogeneous distribution, apparently because of reduced effective population size (Roff 1997). Because species vary in level of heterogeneity in distribution, local populations may more often go extinct in heterogeneously distributed species, resulting in levels of local genetic variation decreasing with increasing heterogeneity in distribution. Lower global population sizes of more heterogeneously distributed species, as we found here, will further contribute to the erosion of genetic variation, as we have shown previously (Møller et al. 2008).

Given that heterogeneously distributed species have small ranges, population sizes, and population densities, low levels of genetic variation, low adult survival rate, low dispersal abilities, and low rates of invasion of urban habitats, why do heterogeneously distributed species not disappear completely? Clearly, heterogeneously distributed species must be superior to more homogeneously distributed species in some respects that can balance the differences documented here. We suggest that heterogeneously distributed species may be locally better competitors than homogeneously distributed species and hence enjoy an advantage. Furthermore, increased dispersal may result in reduced levels of intraspecific competition (e.g., Dall and Cuthill 1997, Clobert et al. 2001), and low local population densities may have a similar effect. Density-dependent predation and parasitism may also contribute to the maintenance of heterogeneously distributed species, because low population densities reduce the impact of predation and the rate of transmission of parasites. We found that species richness of an important group of parasites, the blood parasites that include avian malarial parasites, decreased with increasing heterogeneity in distribution, even when controlling statistically for range size of hosts, body size of hosts and sampling effort. Previously, Soler et al. (2009) reported that the brood parasitic European cuckoo has evolved specific host races mainly in host species with a homogeneous distribution, consistent with this suggestion. Most blood parasites are specialists that are restricted to a single host, or at most a single genus of host (Valkiūnas 2005). The present study extends the findings of Soler et al. (2009) by showing that a homogeneous distribution of hosts is a significant factor contributing to speciation processes. Therefore, heterogeneous distribution of hosts can be considered to be advantageous because it prevents speciation or reduces the rate of speciation by parasites. We hypothesize that heterogeneously distributed species may currently suffer disproportionate declines in range size and population 
size compared to more homogeneously distributed species, even in the absence of a high diversity of parasites, and that this effect may increase during the next decades, when the impact of humans on natural environments is predicted to increase.

In conclusion, we identified an indicator of heterogeneity in spatial distribution that has important ecological implications. Species of passerine birds with heterogeneous distributions as reflected by Morisita's index had low levels of dispersal, and little scope for invasion of novel environments like urban areas. Hence, heterogeneously distributed species had small breeding ranges and small population sizes, suggesting that they might be prone to extinction due to changes in environmental factors, such as human-induced global climatic changes.

\section{ACKNOWLEDGMENTS}

Three anonymous referees provided helpful and constructive comments. The study received financial support from project CGL2007-61251/BOS-FEDER (to J. J. Soler and A. P. Møller) and Junta de Andalucía grants to research groups RNM 340 (to J. J. Soler) and RNM 341 (to M. M. Vivaldi).

\section{Literature Cited}

Aliabadian, M., M. Kaboli, R. Prodon, V. Nijman, and M. Vences. 2006. Phylogeny of Palaearctic wheatears (genus Oenanthe): congruence between morphometric and molecular data. Molecular Phylogenetics and Evolution 42:665-675.

Andrén, H. 1994. Effects of habitat fragmentation on birds and mammals in landscapes with different proportions of suitable habitat: a review. Oikos 71:355-366.

Anscombe, F. J. 1948. The statistical analysis of insect counts based on the negative binomial distribution. Biometrics 5: $165-173$.

Arriero, E., and A. P. Møller. 2008. Host ecology and life history traits associated with blood parasite species richness in birds. Journal of Evolutionary Biology 21:1504-1513.

Becker, W. A. 1984. Manual of quantitative genetics. Academic Enterprises, Pullman, Washington, USA.

Begon, M., J. L. Harper, and C. R. Townsend. 1996. Ecology. Blackwell, Oxford, UK.

Blomqvist, D., M. Andersson, C. Küpper, I. C. Cuthill, J. Kis, R. B. Lanctot, B. K. Sandercock, T. Székely, J. Wallander, and B. Kempenaers. 2002. Genetic similarity between mates and extra-pair parentage in three species of shorebirds. Nature 419:613-615.

Brouat, C., H. Chevallier, S. Meusnier, T. Noblecourt, and J.-Y. Rasplus. 2004. Specialization and habitat: spatial and environmental effects on abundance and genetic diversity of forest generalist and specialist Carabus species. Molecular Ecology 13:1815-1826.

Brown, J. H. 1995. Macroecology. Chicago University Press, Chicago, Illinois, USA.

Brown, J. H., and M. V. Lomolino. 1998. Biogeography. Second edition. Sinauer, Sunderland, Massachusetts, USA.

Clobert, J., J. D. Nichols, E. Danchin, , and A. Dhondt, editors. 2001. Dispersal. Oxford University Press, Oxford, UK.

Cohen, J. 1988. Statistical power analysis for the behavioral sciences. Second edition. Lawrence Erlbaum, Hillsdale, New Jersey, USA.

Combes, C. 2001. Parasitism. The ecology and evolution of intimate interactions. University of Chicago Press, Chicago, Illinois, USA.

Cornell, H. V. 1982. The notion of minimum distance or why rare species are clumped. Oecologia 52:278-280.
Cramp, S., and C. M. Perrins, editors. 1988-1994. The birds of the Western Palearctic. Volumes 5-9. Oxford University Press, Oxford, UK.

Dall, S. R. X., and I. C. Cuthill. 1997. The information costs of generalism. Oikos 80:197-202.

Devictor, V., R. Julliard, and F. Jiguet. 2008. Distribution of specialist and generalist species along spatial gradients of habitat disturbance and fragmentation. Oikos 117:507-514.

Fahrig, L. 1997. Relative effects of habitat loss and fragmentation on population extinction. Journal of Wildlife Management 61:603-610.

Fahrig, L. 2003. Effects of habitat fragmentation on biodiversity. Annual Reviews of Ecology and Systematics 34:487515.

Felsenstein, J. 1985. Phylogenies and the comparative method. American Naturalist 125:1-15.

Futuyma, D. J., and G. Moreno. 1988. The evolution of ecological specialization. Annual Reviews of Ecology and Systematics 19:207-233.

Garland, T., Jr., P. H. Harvey, and A. R. Ives. 1992. Procedures for the analysis of comparative data using phylogenetically independent contrasts. Systematic Biology 41:18-32.

Gaston, K. J. 2003. The structure and dynamics of geographic ranges. Oxford University Press, New York, New York, USA.

Gaston, K. J., and T. M. Blackburn. 1996. Global scale macroecology: interactions between population size, geographic range size and body size in the Anseriformes. Journal of Animal Ecology 65:701-714.

Glutz von Blotzheim, U. N., and K. M. Bauer, editors. 19661997. Handbuch der Vögel Mitteleuropas. Aula-Verlag, Wiesbaden, Germany.

Grell, M. B. 1998. Fuglenes Danmark. Gad, Copenhagen, Denmark.

Hagemeijer, W. J. M., and M. J. Blair. 1997. The EBCC atlas of European breeding birds: their distribution and abundance. T. and A. D. Poyser, London, UK.

Hanski, I. 1991. Single-species metapopulation dynamics: concepts, models and observations. Biological Journal of the Linnean Society 42:17-38.

Hanski, I. 1999. Metapopulation ecology. Oxford University Press, Oxford, UK.

Hanski, I., T. Pakkala, M. Kuussaari, and G. Lei. 1995. Metapopulation persistence of an endangered butterfly in a fragmented landscape. Oikos 72:21-28.

Hartley, S. 1998. A positive relationship between local abundance and regional occupancy is almost inevitable (but not all positive relationships are the same). Journal of Animal Ecology 67:992-994.

Hoelzel, A. R. 1992. Molecular genetic analysis of populations. Oxford University Press, Oxford, UK.

Hurlbert, S. H. 1990. Spatial distribution of the montane unicorn. Oikos 58:257-271.

JMP. 2000. JMP. SAS Institute, Cary, North Carolina, USA.

Jønsson, K. A., and J. Fjeldså. 2006. A phylogenetic supertree of oscine passerine birds (Aves: Passeri). Zoologica Scripta 35:149-186.

Julliard, R., J. Clavel, V. Devictor, F. Jiguet, and D. Couvet. 2006. Spatial segregation of specialists and generalists in bird communities. Ecology Letters 9:1237-1244.

Levins, R. 1962. Theory of fitness in a heterogeneous environment. I. The fitness set and adaptive function. American Naturalist 96:361-373.

Levins, R. 1968. Evolution in changing environments. Princeton University Press, Princeton, New Jersey, USA.

MacArthur, R. H. 1972. Geographical ecology. Harper and Row, New York, New York, USA.

Martí, R., and J. C. del Moral. 2003. Atlas de las aves reproductoras de España. Organismo Autónomo de Parques Nacionales, Madrid, Spain. 
McGarigal, K., and S. A. Cushman. 2002. Comparative evaluation of experimental approaches to the study of habitat fragmentation effects. Ecological Applications 12: 335-345.

Møller, A. P. 2001. Sexual selection, extra-pair paternity, genetic variability and conservation. Acta Zoologica Sinica 47:2-12.

Møller, A. P. 2009. Successful city dwellers: a comparative study of the ecological characteristics of urban birds. Oecologia 159:849-858.

Møller, A. P., P. Christe, and L. Z. Garamszegi. 2005. Coevolutionary arms races: increased host immune defense promotes specialization by avian fleas. Journal of Evolutionary Biology 18:46-59.

Møller, A. P., L. Z. Garamszegi, and C. Spottiswoode. 2008. Genetic similarity, distribution range and sexual selection. Journal of Evolutionary Biology 21:213-225.

Møller, A. P., and M. D. Jennions. 2002. How much variance can be explained by ecologists and evolutionary biologists? Oecologia 132:492-500.

Møller, A. P., and T. A. Mousseau. 2007. Determinants of interspecific variation in population declines of birds from exposure to radiation at Chernobyl. Journal of Applied Ecology 44:909-919.

Møller, A. P., and J. T. Nielsen. 2007. Malaria and risk of predation: a comparative study of birds. Ecology 88:871-881.

Papangelou, A., M. Ham, and M. M. Miyamoto. 1998. Variation of multilocus minisatellite DNA fingerprints in avian populations. Zoological Studies 37:161-168.

Peirce, M. A. 1981. Distribution and host-parasite check-list of the haematozoa of birds in Western Europe. Journal of Natural History 15:419-458.

Purvis, A., and A. Rambaut. 1995. Comparative analysis by independent contrasts (CAIC). Computer and Applied Biosciences 11:247-251.

Reeve, H. K., D. F. Westneat, W. A. Noon, P. W. Sherman, and F. Aquadro. 1990. DNA "fingerprinting" reveals high levels of inbreeding in colonies of the eusocial naked molerat. Proceedings of the National Academy of Science USA 87:2496-2500.

Ricklefs, R., B. L. Swanson, S. Fallon, A. Martínez-Abraín, A. Scheuerlein, J. Gray, and S. C. Latta. 2005. Community relationships of avian malaria parasites in southern Missouri. Ecological Monographs 75:543-559.

Roff, D. A. 1997. Evolutionary quantitative genetics. Chapman and Hall, New York, New York, USA.

Rosenzweig, M. L. 1995. Species diversity in space and time. Cambridge University Press, Cambridge, UK.

Routledge, R. D., and T. B. Swartz. 1991. Taylor's power law re-examined. Oikos 60:107-112.
Saunders, D. A., R. J. Hobbs, and C. R. Margules. 1991. Biological consequences of ecosystem fragmentation: a review. Conservation Biology 5:18-32.

Scheuerlein, A., and R. E. Ricklefs. 2004. Prevalence of blood parasites in European passeriform birds. Proceedings of the Royal Society B 271:1363-1370.

Sibley, C. G., and J. E. Ahlquist. 1990. Phylogeny and classification of birds, a study in molecular evolution. Yale University Press, New Haven, Connecticut, USA.

Slatkin, M. 1987. Gene flow and the geographic structure of natural populations. Science 236:787-792.

Sol, D., S. Timmermans, and L. Lefebvre. 2002. Behavioural flexibility and invasion success in birds. Animal Behaviour 63:495-502.

Soler, J. J., M. Martín Vivaldi, and A. P. Møller. 2009. Geographic distribution of suitable hosts explains the evolution of specialized gentes in the European cuckoo Cuculus canorus. BMC Evolutionary Biology 9:88.

Spottiswoode, C., and A. P. Møller. 2004. Genetic similarity and hatching success in birds. Proceedings of the Royal Society B 271:267-272.

Stattersfield, A. J., and D. R. Capper. 2000. Threatened birds of the World. Lynx Ediciones, Barcelona, Spain.

Taylor, L. R. 1961. Aggregation, variance and the mean. Nature 189:732-735.

Taylor, L. R., I. P. Woiwod, and J. N. Perry. 1978. The densitydependence of spatial behaviour and the rarity of randomness. Journal of Animal Ecology 47:383-406.

Thomas, G. H., M. A. Wills, and T. Székely. 2004. A supertree approach to shorebird phylogeny. BMC Evolutionary Biology 4:28.

Tripet, F., P. Christe, and A. P. Møller. 2002. The importance of host spatial distribution for parasite specialisation and speciation: comparative study of bird fleas (Siphonaptera: Ceratophyllidae). Journal of Animal Ecology 71:735-748.

Tucker, G. M., and M. F. Heath. 1994. Birds in Europe, their conservation status. BirdLife International, Cambridge, UK.

Turner, I. M. 1996. Species loss in fragments of tropical rain forest: a review of the evidence. Journal of Applied Ecology 33:200-209.

Valkiūnas, G. 2005. Avian malaria parasites and other haemosporidia. CRC Press, Boca Raton, Florida, USA.

Waldenström, J., S. Bensch, D. Hasselquist, and Ö. Östman. 2004. A new nested polymerase chain reaction method very efficient in detecting Plasmodium and Haemoproteus infections from avian blood. Journal of Parasitology 90:191-194.

Wetton, J. H., R. E. Carter, D. T. Parkin, and D. Walters. 1987. Demographic study of a wild house sparrow population by DNA fingerprinting. Nature 327:147-149.

Wright, S. 1978. Evolution and the genetics of populations. IV. Variability within and among populations. University of Chicago Press, Chicago, Illinois, USA.

\section{APPENDIX A}

Information on body mass, mean population density, standard deviation in population density, Morisita's index for population density among regions, urbanization, total breeding range, Western Palearctic breeding range, Western Palearctic breeding population size, dispersal distance, annual fecundity, annual adult survival rate, band-sharing coefficient, number of blood parasite species, and number of individuals sampled for blood parasites for 197 bird species from the Western Palearctic (Ecological Archives E091-197-A1).

\section{APPENDIX B}

Phylogenetic relationships among 197 bird species from the Western Palearctic (Ecological Archives E091-197-A2). 